\title{
Linking Libraries to the Web: Linked Data and the Future of the Bibliographic Record
}

\section{Brighid M. Gonzales}

\section{ABSTRACT}

The ideas behind Linked Data and the Semantic Web have recently gained ground and shown the potential to redefine the world of the web. Linked Data could conceivably create a huge database out of the Internet linked by relationships understandable by both humans and machines. The benefits of Linked Data to libraries and their users are potentially great, but so are the many challenges to its implementation. The BIBFRAME Initiative provides the possible framework that will link library resources with the web, bringing them out of their information silos and making them accessible to all users.

\section{INTRODUCTION}

For many years now the MARC (MAchine-Readable Cataloging) format has been the focus of rampant criticisms across library-related literature, and though an increasing number of diverse metadata formats for libraries, archives, and museums have been developed, no framework has shown the potential to be a viable replacement for the long-established and widely used bibliographic format. Over the past decade, web technologies have been advancing at a progressively rapid pace, outpacing MARC's ability to keep up with the potential these technologies can offer to libraries. Standing by the MARC format leaves libraries in danger of not being adequately prepared to meet the needs of modern users in the information environments they currently frequent (increasingly, search engines such as Google).

New technological developments such as the ideas behind Linked Data and the Semantic Web have the potential to bring a host of benefits to libraries and other cultural institutions by allowing libraries and their carefully cultivated resources to connect with users on the web. Though there remains a host of obstacles to their implementation, Linked Data has much to offer libraries if they can find ways to leverage this technology for their own uses. Libraries are slowly finding ways to take advantage of the opportunities Linked Data present, including initiatives such as the Bibliographic Framework Initiative, known as BIBFRAME, which may have the potential to be the bibliographic replacement for MARC that the information community has long needed. Such a change may help libraries not only to stay current with the modern information world and stay relevant in the minds of users, but also reciprocally create a richer world of data available to information seekers on the web.

Brighid Gonzales (brighidmgonzales@gmail.com), a recent MLIS recipient from the School of Library and Information Science, San Jose State University, is winner of the 2014 LITA/Ex Libris Student Writing Award. 


\section{The Limitations of MARC}

Much has been written over the years about the issues and shortcomings of the MARC format. Nonetheless, MARC formatting has been widely used by libraries around the world since the 1960s, when it was first created. This long-established and ubiquitous usage has resulted in countless legacy bibliographic records that currently exist in the MARC format. To lose this carefully crafted data or to expend the finances, time, and manual effort required to convert all of this legacy data into a new format may be a cause for reservation in the community.

But the fact remains that in spite of its widespread use, there are many issues with the MARC format that make it a candidate for replacement in the world of bibliographic data.

Andresen describes several different versions of MARC that have largely been wrapped together in the community's mind, reminding us that "although MARC21 is often described as an international standard, it is only used in a limited number of countries." ${ }^{1}$ In actuality, what we often refer to simply as MARC could be MARC21, UKMARC, UNIMARC or even danMARC2. ${ }^{2}$ This lack of a unified standard has long been an issue with this particular format.

Then there is MARC's notorious inflexibility. Originally created for the description of printed materials, MARC's rigidly defined standards can make it unsuited for the description of digital, visual, or multimedia resources. Andresen writes that "the lack of flexibility means that local additions might hinder exchange between local systems and union catalogue systems." ${ }^{3}$ Tennant has also expressed frustration with MARC's inflexibility, particularly its inability to express hierarchical relationships. Tennant posits that where the MARC format is "flat," expressing relationships involving hierarchy, such as in a table of contents, "would be a breeze in XML," which is the format he recommends moving toward for its greater extensibility. ${ }^{4}$ MARC's rigidity may also be a reason why the format is not generally used outside of the library environment; thus information contained in MARC format cannot be exchanged with information from nonlibrary environments. ${ }^{5}$

Inconsistencies, errors, and localized practices are also issues frequently cited in detailing MARC's inherent shortcomings. With shared cataloging, inconsistencies may be less common, but there remains the fact that with any number of individual catalogers creating records, the potential for error is still great. And any localized changes can also create inconsistency in records from library to library. Tennant gives as an example recording the editor of a book, which "should be encoded in a 700 field, with a \$e subfield that specifies the person is the editor. But the \$e subfield is frequently not encoded, thus leaving one to guess the role of the person encoded in the 700 field." 6

When it comes to issues with MARC in the modern computing environment, however, one of the biggest and seemingly insurmountable problems is its inability to express the relationships between entities. Andresen points out that it is "difficult to handle relations between data that are described in different fields,"7 while Tennant writes that "relationships among related titles are problematic."8 Alemu et al. also write of MARC's "document-centric" structure, which prevents it 
from recognizing relationships between entities that might be possible in a more "actionable datacentric format." 9

Though Tennant advocates the embrace of XML-based formats as a way to transition from MARC, Breeding writes that even MARCXML "cannot fully make intelligible the quirky MARC coding in terms of semantic relationships." ${ }^{10}$ Alemu et al. also note that MARC may continue to be widely used mainly because alternatives, including XML, have not yet been found to be an adequate replacement. ${ }^{11}$

It is clear that if libraries and their carefully crafted bibliographic records are to remain relevant and viable in today's modern computing world, a more modern metadata format that addresses these issues will be required. Clearly needed is a more flexible and extensible format that allows for the expression of relationships between points of data and the ability to link that data to other related information outside of the presently insular library catalog.

\section{Linked Data and the Semantic Web}

Linked Data works as the framework behind the Semantic Web, an idea by World Wide Web inventor Tim Berners-Lee, which would turn the Internet into something closer to one large database rather than simply a disparate collection of documents. Since the Internet is often the first place users turn to for information, libraries should take advantage of the concepts behind Linked Data to both put their resources out on the web, where they can be found by users, and in turn bring those users back to the library through the lure of authoritative, high-quality resources.

In the world of Linked Data, the relationships between data, not just the documents in which they are contained, are made explicit and readable by both humans and machines. With the ability to "understand" and interpret these semantically explicit connections, computers will have the power to lead users to a web of related data based on a single information search. Underpinning the Semantic Web are the web-specific standards XML and RDF (Resource Description Framework). These work as universal languages for semantically labeling data in such a way that both a person and a computer can interpret their meaning and then distinguishing the relationships between the various data sources.

These relationships are expressed using RDF, "a flexible standard proposed by the W3C to characterize semantically both resources and the relationships which hold between them."12 Baker notes that RDF supports "the process of connecting dots—of creating "knowledge" - by providing a linguistic basis for expressing and linking data."13 RDF is organized into triples, expressing meaning as subject, verb, and object and detailing the relationships between them. An example is The Catcher in the Rye is written by J. D. Salinger, where The Catcher in the Rye acts as the subject, J. $D$. Salinger is the object and the "verb" is written by expresses the semantic relationship between the two, naming J. D. Salinger as the author of The Catcher in the Rye. By using this framework, computers can link to other RDF-encoded data, leading users to other works written by J. D. Salinger, other adaptations of The Catcher in the Rye, and other related data sources from around 
the web.

RDF gives machines the ability to "understand" the semantic meaning of things on the web and the nature of the relationships between them. In this way it can make connections for people, leading them to related information they may not have otherwise found. The use of XML allows developers to create their own tags, adding an explicit semantic structure to their documents that they can exploited using RDF.

The Semantic Web is based on four rules explicated by web inventor Tim Berners-Lee. The rules for the Semantic Web are as follows:

1. Use URIs (uniform resource identifiers) as names for things.

2. Use HTTP URIs so that people can look up those names.

3. When someone looks up a URI, provide useful information, using the standards (RDF*, SPARQL).

4. Include links to other URIs so that they can discover more things. ${ }^{14}$

URIs act as a permanent signpost for things, both on and off the web. Using consistent URIs allows data to be linked between and back to certain places on the web without the worry of broken or dead links. RDF triples map the relationships between each thing, which can then be linked to more things, opening up a wide world of interrelated data for users.

The concept behind Linked Data would allow for the integration of library data and data from other resources, whether from "scientific research, government data, commercial information, or even data that has been crowd-sourced." 15 However, to create an open web of data facilitated by Linked Data theories, open standards such as RDF must be used, making data interoperable with resources from various communities. This interoperability is key to being able to mix library resources with those from other parts of the web.

Interoperability helps to make "data accessible and available, so that they can be processed by machines to allow their integration and their reuse in different applications." 16 In this way, machines would be able to understand the relationships and connections between data contained within documents and thus lead users to related data they may not have otherwise found. Using Linked Data would bring carefully crafted and curated library data out of the information silos in which they have long been enclosed and connect them with the rest of the web where users can more easily find them.

\section{Benefits For Libraries}

Libraries and their users have much to gain from participation in the Linked Data movement. In an age when Google is often the first place users turn when searching for information, freeing library data from their insulated databases and getting them out onto the web where the users are can help make library resources both relevant and available for users who may not make the library 
the first place they look for information. This can lead not only to increased use by library patrons and nonpatrons (who would now be potential library patrons) alike, but also to increased visibility for the library. Creating and using Linked Data technologies also opens the door for libraries to share metadata and other information in a way previously limited by MARC. Libraries also have the potential to add to the richness of data that is available on the web, creating a reciprocal benefit with the Semantic Web itself.

Coyle writes that "every minute an untold number of new resources is added to our digital culture, and none of these is under the bibliographic control of the library." ${ }^{17}$ Indeed, the World Wide Web is a participatory environment where anyone can create, edit or manipulate information resources. Libraries still consider themselves the province of quality, reliable information, but users don't necessarily go to libraries when searching and don't necessarily have the Internet acumen to distinguish between authoritative information and questionable resources. Coyle also notes that "the push to move libraries in the direction of linked data is not just a desire to modernize the library catalog; it represents the necessity to transform the library catalog from a separate, closed database to an integration with the technology that people use for research."18 Using Linked Data, libraries can still create the rich, reliable, authoritative data they are known for while also making it available on the web, where potentially anyone can find it.

Much has been written about libraries' information silos, and many researchers are finding in Linked Data the possibility to free this information. For the information contained in the library catalog to be significantly more usable it "must be integrated into the web, queryable from it, able to speak and to understand the language of the web."19 Alemu et al. write that linking library data to the web "would allow users to navigate seamlessly between disparate library databases and external information providers such as other libraries, and search engines." ${ }^{20}$ Users are likely to find the world of Linked Data immeasurably more useful than individually searching library databases one-by-one or relying on Google search results for the information they need.

Linked Data also allows for the possibility of serendipity in information searching, of finding information one didn't even know they were looking for, something akin to browsing the library shelves. ${ }^{21}$ Linked Data "allows for the richer contextualization of sources by making connections not only within collections but also to relevant outside sources."22 Tillett adds that Linked Data would allow for "mashups and pathways to related information that may be of interest to the Web searcher-either through showing them added facets they may wish to consider to refine their search or suggesting new directions or related resources they may also like to see."23

The use of Linked Data is not just beneficial to users though. Libraries are also likely to see increased benefits in the sharing of metadata and other resources. Alemu et al. write that "making library metadata available for re-use would eliminate unnecessary duplication of data that is already available elsewhere, through reliable sources. ${ }^{24}$ Tillett also writes about the reduced cost to libraries for storage and data in a linked data environment where "libraries do not need to replicate the same data over and over, but instead share it mutually with each other and with 
others using the Web," reducing costs and expanding information accessibility. ${ }^{25}$ Byrne and Goddard also note that "having a common format for all data would be a huge boon for interoperability and the integration of all kinds of systems."26

In addition to the reduced cost of shared resources, something with which libraries are already very familiar, the linking of data from libraries to one another and to the web would also allow for an increased richness in overall data. From metadata that may need to be changed or updated periodically to user-generated metadata that is more likely to include current, up-to-date terminology, the "mixed metadata" approach allowed by Linked Data would be "better situated to provide a richer and more complete" description of various resources that could more accurately provide for the variety of interpretation and terminology possible in their description. ${ }^{27}$

\section{A New Bibliographic Framework}

One of the most important ways libraries are moving toward the world of Linked Data is with the Bibliographic Framework Initiative, known as BIBFRAME, which was announced by the Library of Congress in 2011. Since then, though BIBFRAME is still in development, rapid progress has been made that suggests that BIBFRAME may be the long-awaited replacement for the MARC format that could free library bibliographic information from its information silos and allow it to be integrated with the wider web of data.

The BIBFRAME model comprises four classes: Creative Work, Instance, Authority, and Annotation. In this model, Creative Work represents the "conceptual essence" of the item. Instance is the "material embodiment" of the Creative Work. Authority is a resource that defines relationships reflected by the Creative Work and Instance, such as People, Places, Topics, and Organizations. Annotation relates the Creative Work with other information resources, which could be library holdings information, cover art, or reviews. ${ }^{28}$ These are similar in a way to the FRBR (Functional Requirements for Bibliographic Records) model, which uses Work, Expression, Manifestation, and Item. ${ }^{29}$ Indeed, BIBFRAME is built with RDA (Resource Description and Access) as an important source for content, which was in turn built around the principles in FRBR. Despite this, BIBFRAME "aims to be independent of any particular set of cataloging rules."30

Realizing the vast amounts of information that is still recorded in MARC format, the BIBFRAME initiative is also working on a variety of tools that will help to transform legacy MARC records into BIBFRAME resources. ${ }^{31}$ These tools will be essential as "the conversion of MARC records to useable Linked Data is a complicated process." ${ }^{2}$ Where MARC allowed for libraries to share bibliographic records without each having to constantly reinvent the wheel, BIBFRAME will allow library metadata to be "shared and reused without being transported and replicated." 33

BIBFRAME would support the Linked Data model while also incorporating emerging content standards such as FRBR and RDA. ${ }^{34}$ The BIBFRAME initiative is committed to compatibility with existing MARC records but would eventually replace MARC as a bibliographic framework "agnostic to cataloging rules" 35 rather than intertwined with them as MARC was with AACR2. Also unlike 
MARC, which is rigidly structured and not amenable to incorporation with web standards, BIBFRAME would enable library metadata to be found on the web, freeing it from the information silos that have contained it for decades. Whereas MARC is not very web-compatible, "BIBFRAME is built on XML and RDF, both 'native' schemas for the internet. The web-friendly nature of these schemas allows for the widest possible indexing and exposure for the resources held in libraries." 36

Backed by the Library of Congress, BIBFRAME already has a great deal of support throughout the information community, though it is not yet at the stage of implementation for most libraries. However, half a dozen libraries and other institutions are acting as "Early Experimenters" working to implement and experiment with BIBFRAME to assist in the development process and get the framework library ready. Participating institutions include the British Library, George Washington University, Princeton University, Deutsche National Bibliothek, National Library of Medicine, OCLC, and the Library of Congress. ${ }^{37}$ Though not yet fully realized, BIBFRAME seems to offer a substantial step toward the implementation of Linked Data to connect library bibliographic materials with other resources on the web.

\section{The Challenges Ahead}

The road to widespread use of the Semantic Web, Linked Data, and even possible implementations such as BIBFRAME is not without obstacles. For one, knowledge and awareness is a major concern, as well as the intimidating thought of transitioning away from MARC, a standard that has been in widespread use for as long as many of the professionals using it have been alive. There is also the challenge and significant resources required for converting huge stores of legacy data from MARC format to a new standard. In addition, Linked Data has its own set of specific concerns, such as legality and copyright issues involved in the sharing of information resources, as well as the willingness of institutions to share metadata that they may have invested a great deal of time and money in creating.

Many organizations may be hesitant to make the move toward Linked Data without a clear sign of success from other institutions. Chudnov writes that "a new era of information access where library-provided resources and services rose swiftly to the top of ambient search engines' results and stayed there" is what may be necessary, as well as "tools and techniques that make it easier to put content online and keep it there."38 Byrne and Goddard also note that "Linked Data becomes more powerful the more of it there is. Until there is enough linking between collections and imaginative uses of data collections there is a danger librarians will see linked data as simply another metadata standard, rather than the powerful discovery tool it will underpin."39

Alemu et al. concur that making Linked Data easy to create and put online is necessary before potential implementers will begin to use it. "It is imperative that the said technologies be made relatively easy to learn and use, analogous to the simplicity of creating HTML pages during the early days of the web." 40 The potential learning curve involved in Linked Data may be a great barrier to its potential use. Tennant writes in an article about moving away from MARC to a more 
modern bibliographic framework that users "must dramatically expand our understanding of what it means to have a modern bibliographic infrastructure, which will clearly require sweeping professional learning and retooling." ${ }^{41}$

Even without considering ease-of-use difficulties or the challenges in teaching practitioners an entirely new bibliographic system, the fact remains that transitioning away from MARC toward any new bibliographic infrastructure system will require a great deal of resources, time and effort. "There are literally billions of records in MARC formats; an attempt at making the slightest move away from it would have huge implications in terms of resources." 42 Breeding also writes of the potential trauma involved in shifting away from MARC, which is currently integral to many library automation systems. ${ }^{43} \mathrm{~A}$ shift to anything else would require not just the cooperation of libraries but also of vendors, who may see no reason to create systems compatible with anything other than MARC. As Tennant writes, "Anyone who has ever been involved with migrating from one integrated library system to another knows, even moving from one system based on MARC/AACR2 to another can be daunting." 44 Moving from a MARC/AACR2-based system to one based on an entirely new framework may be more of a challenge than many libraries would like to take on.

A move to something such as BIBFRAME may be fraught with even more difficulty, though it is impossible to say before such an implementation has been fully realized. Library system software is not yet compatible with BIBFRAME, and as Kroeger writes, "Most libraries will not be able to implement BIBFRAME because their systems do not support it, and software vendors have little incentive to develop BIBFRAME integrated library systems without reasonable certainty of library implementation of BIBFRAME." 45 This catch-22 situation may be difficult to remedy without a large cooperative effort between libraries, vendors, and the entire information community.

Another potential obstacle to BIBFRAME implementation that Kroeger suggests is the possible difficulty in providing interoperability with all of the many other metadata standards currently in existence. ${ }^{46}$ This is an issue that Tennant also considers in his recommendations that a new bibliographic infrastructure compatible with modern library and information needs must be versatile, extensible, and especially interoperable with other metadata schemes currently in use. ${ }^{47}$ XML has proven to be useful for a wide variety of metadata schemas, but BIBFRAME would need to be able to make library data held in a huge variety of metadata standards available for use on the web.

Another issue, cited by Byrne and Goddard, is that of privacy. "Librarians, with their long tradition of protecting the privacy of patrons, will have to take an active role in linked data development to ensure rights are protected."48 Issues of copyright and ownership, something libraries already grapple with in the licensing of various library journals, databases, and other electronic resources, may be insurmountable. "Libraries no longer own much of the content they provide to users; rather it is subscribed to from a variety of vendors. Not only does that mean that vendors will have to make their data available in linked data formats for improvements to federated search to happen, but a mix of licensed and free content in a linked data environment would be extremely 
difficult to manage." 49 Again, overcoming obstacles such as these would require intense negotiation and cooperation between libraries and vendors. A sustainable and viable move to a Linked Data environment would need to be a cooperative effort between all involved parties and would have to have the full support and commitment of everyone involved before it could begin to move forward.

\section{Moving Libraries toward Linked Data}

Making the move toward the use of Linked Data and modern bibliographic implementations such as BIBFRAME will require a great deal of cooperation, sharing, learning, and investigation, but libraries are already starting to look toward a linked future and what it will take to get there. Libraries will need to begin incorporating the principles of Linked Open Data in their own catalogs and online resources as well as publishing and sharing as much data as possible. Libraries also need to put forth a concerted effort to encourage vendors to move toward library systems which can accommodate a linked data environment.

Alemu et al. write that cooperation and collaboration between all of the involved stakeholders will be a crucial piece to the transfer of library metadata from catalog to web. In the process, and as part of this cooperative effort, libraries will have to wholeheartedly adopt the RDF/XML format, something Alemu et al. deem "mandatory." 50 This would support the "conceptual shift from perceiving library metadata as a document or record to what Coyle (2010) terms as actionable metadata, i.e., one that is machine-readable, mash-able and re-combinable metadata." ${ }^{51}$

Chudnov adds that libraries will need to follow "steady URL patterns" for as much of their resources as possible, one of the key rules of Linked Data. ${ }^{22} \mathrm{He}$ also notes that we will know we have made progress on the implementation of Linked Data when "link hubs at smaller libraries (aka catalogs and discovery systems) cross link between local holdings, authorities, these national authority files, and peer libraries that hold related items," though the real breakthrough will come when "the big national hubs add reciprocal links back out to smaller hub sites." 53 Before this can happen, however, libraries must make sure that all of their own holdings link to each other, from the catalog to items in online exhibits. Chudnov also advocates adding user-generated knowledge into the mix by allowing users to make new connections between resources when and where they can. ${ }^{54}$

Borst, Fingerle, and Neubert, in their conference report from 2009, write that libraries and projects using linked data need to regard the catalog as a network, publish their data as Linked Data using the Semantic Web standards laid out by Tim Berners-Lee, and link to external URIs. ${ }^{55}$ They also suggest libraries use and help to further develop open standards that are already available rather than rely on in-house developments. ${ }^{56}$ In their final recommendation, they write that while libraries need to publish their data as open Linked Data on the web, they should also try to do so with the "least possible restrictions imposed by licences in order to ensure widest reusability." 57 


\section{CONCLUSION}

The theories behind Linked Data and the Semantic Web are still in the process of being drawn out, but it is clear that at this point they are more than hypotheticals. Linked Data is the possible future of the web and how information will be organized, searched for, discovered, and retrieved. As search algorithms continue to improve and users continue to turn to them first (and sometimes entirely) for their information needs, libraries will need to make major changes to ensure the data they have painstaking created and curated over the decades remains relevant and reachable to users on the web. Linked Data provides the opportunity for libraries to integrate their authoritative data with user-generated data from the web, creating a rich network of reliable, current, far-reaching resources that will meet users' needs wherever they are.

Libraries have always been known to embrace technology to stay at the forefront of user needs and provide unique and irreplaceable user services. To stay current with shifts in modern technology and user behavior, libraries need to be a driving force in the implementation of Linked Data, embrace Semantic Web standards, and take full advantage of the benefits and opportunities they present. Ultimately, libraries can leverage the advantages created by Linked Data to construct a better information experience for users, keeping libraries both a relevant and more highly valued part of information retrieval in the twenty-first century.

\section{REFERENCES}

1. Leif Andresen, "After MARC—What Then?" Library Hi Tech 22, no. 1 (2004): 41.

2. Ibid., 40-51.

3. Ibid., 43.

4. Roy Tennant, “MARC Must Die," Library Journal 127, no. 17 (2002): 26-28, http://lj.libraryjournal.com/2002/10/ljarchives/marc-must-die/\#_.

5. Andresen, "After MARC-What Then?"

6. Tenant, "MARC Must Die."

7. Andresen, "After MARC-What Then?", 43.

8. Tenant, "MARC Must Die."

9. Getaneh Alemu et al., "Linked Data for Libraries: Benefits of a Conceptual Shift From LibrarySpecific Record Structures to RDF-based Data Models," New Library World 113, no. 11/12 (2012): 549-570, http://dx.doi.org/10.1108/03074801211282920.

10. Marshall Breeding, "Linked Data: The Next Big Wave or Another Tech Fad?," Computers in Libraries 33, no. 3 (2013): 20-22, http://www.infotoday.com/cilmag/.

11. Alemu et al., "Linked Data for Libraries." 
12. Mauro Guerrini and Tiziana Possemato, "Linked Data: A New Alphabet for the Semantic Web," Italian Journal of Library \& Information Science 4, no. 1 (2013): 79-80, http://dx.doi.org/10.4403/jlis.it-6305.

13. Tom Baker, "Designing Data for the Open World of the Web," Italian Journal of Library \& Information Science 4, no 1 (2013): 64, http://dx.doi.org/10.4403/jlis.it-6308.

14. Tim Berners-Lee, "Linked Data," W3.org, last modified June 18, 2009, http://www.w3.org/DesignIssues/LinkedData.html.

15. Karen Coyle, "Library Linked Data: An Evolution," Italian Journal of Library \& Information Science 4, no 1 (2013): 58, http://dx.doi.org/10.4403/jlis.it-5443.

16. Gianfranco Crupi, "Beyond the Pillars of Hercules: Linked Data and Cultural Heritage," Italian Journal of Library \& Information Science 4, no. 1 (2013), 36, http://dx.doi.org/10.4403/jlis.it8587.

17. Coyle, "Library Linked Data: An Evolution," 56.

18. Ibid., 56-57.

19. Crupi, "Beyond the Pillars of Hercules," 35.

20. Alemu et al., "Linked Data for Libraries," 562.

21. Ibid.

22. Thea Lindquistet al., "Using Linked Open Data to Enhance Subject Access in Online Primary Sources," Cataloging \& Classification Quarterly 51 (2013): 913-928, http://dx.doi.org/10.1080/01639374.2013.823583.

23. Barbara Tillett, "RDA and the Semantic Web, Linked Data Environment," Italian Journal of Library \& Information Science 4, no. 1 (2013): 140, http://dx.doi.org/10.4403/jlis.it-6303.

24. Alemu et al., "Linked Data for Libraries."

25. Tillett, "RDA and the Semantic Web, Linked Data Environment," 140.

26. Gillian Byrne and Lisa Goddard, "The Strongest Link: Libraries and Linked Data," D-Lib Magazine 16, no. 11/12 (2010), http://dx.doi.org/10.1045/november2010-byrne.

27. Alemu et al., "Linked Data for Libraries," 560.

28. Library of Congress, Bibliographic Framework as a Web of Data: Linked Data Model and Supporting Services, (Washington, DC: Library of Congress, November 21 2012), http://www.loc.gov/bibframe/pdf/marcld-report-11-21-2012.pdf.

29. Barbara Tillett, "What is FRBR? A Conceptual Model for the Bibliographic Universe," Library of 
Congress, 2003, http://www.loc.gov/cds/downloads/FRBR.PDF.

30. "BIBFRAME Frequently Asked Questions," Library of Congress, http://www.loc.gov/bibframe/faqs/\#q04.

31. Ibid.

32. Lindquist et al., "Using Linked Open Data to Enhance Subject Access in Online Primary Sources," 923.

33. Alan Danskin, "Linked and Open Data: RDA and Bibliographic Control." Italian Journal of Library \& Information Science 4, no. 1 (2013): 157, http://dx.doi.org/10.4403/jlis.it-5463.

34. Erik T. Mitchell, "Three Case Studies in Linked Open Data." Library Technology Reports 49, no. 5 (2013): 26-43. http://www.alatechsource.org/taxonomy/term/106.

35. Angela Kroeger, "The Road to BIBFRAME: The Evolution of the Idea of Bibliographic Transition into a Post MARC Future," Cataloging \& Classification Quarterly 51 (2013): 881, http://dx.doi.org/10.1080/01639374.2013.823584.

36. Jason W. Dean, "Charles A. Cutter and Edward Tufte: Coming to a Library near You, via BIBFRAME," In the Library with the Lead Pipe, December 4, 2013, http://www.inthelibrarywiththeleadpipe.org/2013/charles-a-cutter-and-edward-tuftecoming-to-a-library-near-you-via-bibframe/.

37. "BIBFRAME Frequently Asked Questions," Library of Congress, http://www.loc.gov/bibframe/faqs/\#q04.

38. Daniel Chudnov, "What Linked Data Is Missing," Computers in Libraries 31, no. 8 (2011): 3536,http://www.infotoday.com/cilmag.

39. Byrne and Goddard, "The Strongest Link: Libraries and Linked Data."

40. Alemu et al., "Linked Data for Libraries," 557.

41. Roy Tennant, "A Bibliographic Metadata Infrastructure for the Twenty-First Century," Library Hi Tech 22, no. 2 (2004): 175-181, http://dx.doi.org/10.1108/07378830410524602.

42. Alemu et al., "Linked Data for Libraries," 556.

43. Breeding, "Linked Data."

44. Tennant, "A Bibliographic Metadata Infrastructure for the Twenty-First Century."

45. Kroeger, "The Road to BIBFRAME," 884-885.

46. Ibid.

47. Tennant, "A Bibliographic Metadata Infrastructure for the Twenty-First Century." 
48. Byrne and Goddard, "The Strongest Link: Libraries and Linked Data.

49. Ibid.

50. Alemu et al., "Linked Data for Libraries."

51. Ibid., 563.

52. Chudnov, "What Linked Data is Missing."

53. Ibid.

54. Ibid.

55. Timo Borst, Birgit Fingerle, and Joachim Neubert, "How Do Libraries Find Their Way onto the Semantic Web?" Liber Quarterly 19, no 3/4 (2010): 336-43, http://liber.library.uu.nl/index.php/lq/article/view/7970/8271.

56. Ibid.

57. Ibid., 342-343. 